\title{
CONTEXTOS, CONTINGENCIA E INTERESES EN EL PROCESO DE INVESTIGAR LA FORMACIÓN DOCENTE. UN ESTUDIO DE CASOS $^{1}$
}

\section{Tatiana Cisternas León ${ }^{2}$}

\begin{abstract}
RESUMEN
Asumiendo que el proceso de investigar supone decisiones enmarcadas en un tiempo y espacio, se analiza cómo los investigadores delimitan sus objetos de estudio en el campo de la formación docente. La metodología, basada en la Teoría Fundamentada, utiliza entrevistas a investigadores para reconstruir la génesis de sus estudios. Los hallazgos muestran orígenes teóricos y pragmáticos que subyacen en las prácticas investigativas. El peso de la posición del investigador en el campo de la formación e investigación, el papel de las convocatorias a concurso (como un artefacto definidor de problemas), los efectos del financiamiento y el protagonismo de la institución que acoge al investigador. Se concluye que la investigación en esta área del campo educativo es un proceso constructivo que involucra factores y decisiones personales, institucionales y contextuales.
\end{abstract}

Palabras clave: Procesos de investigación, producción de conocimiento, estudios sociales de la ciencia, investigación educativa, formación docente.

\section{CONTEXTS, EVENTUALITY AND INTERESTS IN THE RESEARCH PROCESS ON TEACHING EDUCATION: STUDY OF CASES}

\section{ABSTRACT}

Assuming that the research process involves decisions framed in time and space, we analyze how researchers define their objects of study in the field of teacher education. The methodology, based on backed up theory, has used interviews to researchers to reconstruct the genesis of their studies. The findings show theoretical and pragmatic origins underlying research practices: the weight of the position of the researcher in the field of training and research, the role of the calls for competition (as an «artifact» defining problems), the effects of financing and the role of the institution hosting the research. It is concluded that research on education is a constructive process that involves personal, institutional and contextual decisions and factors.

Keywords: Research processes, production of knowledge, social studies of science, research education, teacher education

1 Estudio financiado por Conicyt a través de la Beca de Formación de Doctorado: Investigación sobre formación docente en Chile. Una aproximación a partir de la construcción de sus objetos de estudio.

2 Licenciada en Educación, Doctora en Ciencias de la Educación. Centro de Investigación y Desarrollo en Educación (CIDE), Facultad de Educación, Universidad Alberto Hurtado, Santiago, Chile. Contacto: tcistern@uahurtado.cl 


\section{CONTEXTOS, CONTINGENCIA E INTERESES EN EL PROCESO DE INVESTIGAR LA FORMACIÓN DOCENTE. UN ESTUDIO DE CASOS}

\section{Introducción}

Este artículo presenta parte de los hallazgos de un trabajo de investigación doctoral ${ }^{3}$ en el cual se exploran en profundidad los procesos de investigación en el campo de la formación docente a través del análisis de las condiciones que dieron origen específicamente a nueve investigaciones.

Antecedentes que provienen de los estudios sociales de la ciencia y de revisiones críticas realizadas al campo de la investigación educativa refuerzan la tesis que se desarrollará a lo largo de este artículo: no es suficiente con reflexionar acerca de qué y de cómo investigamos (Cisternas, 2011), sino que también debemos preguntarnos por qué investigamos lo que investigamos. Esto supone comprender la investigación como una práctica social, situada en un tiempo y en un espacio determinado (y de alguna manera determinante), cargada de decisiones y sujeta a intereses donde sus productos (las investigaciones) llevan las huellas de este marco en el que se producen.

En efecto, si los estudios relacionados con la práctica de investigar muestran evidencias respecto de la importancia que tiene el contexto y la contingencia en el proceso de decidir qué investigar, nos preguntamos entonces qué procesos y dimensiones intervienen en la

3 Tesis para optar al grado académico de Doctora en Ciencias de la Educación: Investigación sobre formación docente en Chile. Una aproximación a partir de la construcción de sus objetos de estudio. Pontificia Universidad Católica de Chile. 
definición de los objetos de estudio ${ }^{4}$ que abordan las investigaciones acerca de la formación docente en nuestro país: ¿cómo se construyen esos objetos de investigación en torno a la formación docente?, ¿cuáles son las condiciones que subyacen en la definición de unos focos particulares de preocupación?, ¿qué contextos y contingencias participan en la definición de los objetos de estudio?, ¿cuál es el papel que juega la posición del investigador y los contextos donde investiga?, ¿hay otras condiciones que influyan en la definición de focos problemáticos?

En el primer apartado de este artículo presentamos antecedentes relevantes para el estudio y la perspectiva teórica desde donde se abordan estas preguntas. En seguida describimos las principales decisiones metodológicas y las características de los casos estudiados. Finalmente, desarrollamos los principales hallazgos y las conclusiones del estudio.

\section{Marco conceptual. El carácter constructivo y situacional del proceso de investigar}

¿Qué factores están vinculados con las decisiones que toma el investigador respecto de aquello que investiga?, ¿cómo es el proceso de delimitación de un área de interés, transformación en un objeto de estudio y luego en producto de investigación? Son escasos los estudios que aborden estas preguntas en torno a la producción de conocimiento en el campo educativo, sin embargo, desde otras áreas este fenómeno ha sido ampliamente estudiado y conceptualizado. A continuación algunos aportes que sirvieron de marco para esta investigación.

En los últimos años la investigación ha sido objeto de estudio para varias disciplinas vinculadas con las ciencias sociales (filosofía,

4 Sostenemos que tanto las preguntas que orientan al investigador como los objetivos que define en la investigación son parte de lo que aquí denominamos como «objeto de estudio». Por ello, el lector podrá advertir que al referirnos a los objetos de estudio incluimos las preguntas o problemas que define el investigador, entendido como un foco que se puede identificar en dicha problematización. Así, nos referiremos al mismo núcleo conceptual cuando mencionemos la idea de «objeto» «foco problemático»o «preguntas de investigación». 
historia, antropología y sociología, entre otras) desde la premisa de que el ejercicio de investigar no es una tarea de sujetos aislados, sino que implica siempre una acción conjunta. Solo luego de la primera mitad del siglo XX, la ciencia en sus dimensiones institucionales, cognitivas, prácticas, culturales y sociales se convirtió en un campo de investigación reconocido. El carácter reciente de este desarrollo puede parecer sorprendente habida cuenta del importante espacio que ocupa la ciencia en nuestras sociedades, en especial a partir del siglo XIX. El freno para que emergieran estudios respecto del proceso de investigar era, de hecho, poderoso: un cientificismo que veía en la ciencia una actividad particular, diferente de las demás actividades humanas y protegida de las influencias sociales.

Un cambio en los marcos de análisis al proceso real de investigación hace surgir nuevas concepciones acerca de hacer investigación (Bourdieu, 2003; Knorr-Cetina, 2005; Latour y Woolgar, 1995). A este conjunto de aportes se les ha denominado estudios sociales de la ciencia y si bien presentan diferencias entre sí (GESCIT, 2007; Ramos, 2008) todos ellos coinciden al relevar la importancia que tiene el contexto, la contingencia, los intereses y la posición del investigador en el proceso de decidir qué investigar. Además, reconocen la necesidad de realizar estudios de este tipo para comprender mejor cuáles son las dimensiones involucradas en la construcción social e histórica de los conocimientos que se producen al alero de distintas disciplinas (Ramos, 2008).

La literatura más interesante para efectos de este trabajo refleja que los resultados de la investigación (los nuevos conocimientos que se hacen circular en forma de artículos, informes, presentaciones en congresos, libros, etc.) son el producto de una práctica constructiva, que supone una cadena de decisiones siempre enmarcadas en un tiempo y un espacio particular (Knorr-Cetina, 2005) e impulsadas por intereses diversos que llevan a los investigadores a realizar traducciones particulares respecto de problemas comunes (Latour, 1990). Asimismo, la elección de un tema de investigación y muchas de las decisiones subsiguientes se asocian con la posición en el campo de estudio y con las disposiciones (habitus) del investigador (Bourdieu, 2003). Revisaremos por separado estas dos ideas. 
En primer término, la corriente denominada antropología de las prácticas científicas donde encontramos los trabajos de Knorr-Cetina (2005), Latour y Woolgar (1995) propone estudiar la vida cotidiana de los investigadores en los laboratorios donde ellos hacen investigación. De allí concluyen que los resultados de las investigaciones están ligados fundamentalmente a sus particulares condiciones de producción. Vale decir, están situados local y temporalmente, experimentando las que serían sus propias circunstancias (Martin, 2003). En efecto, el tiempo y el espacio recuperados en las prácticas de investigación suponen asumirlos desde un punto de vista constructivo más que descriptivo: «los productos de la ciencia son construcciones contextualmente específicas que llevan las marcas de la contingencia situacional y de la estructura de intereses del proceso por el cual son generados» (Knorr-Cetina, 2005: 60-61). Siguiendo a esta autora, si lo que queremos es comprender esos productos (en nuestro caso, lo que se investiga acerca de la formación de profesores en nuestro país) es necesario analizar su proceso de construcción. Es decir, la carga de decisiones que enmarcaría el origen de una investigación: «los productos de la ciencia están estructurados internamente por medio de lo que ocurre en el proceso de producción» (Knorr-Cetina, 2005: 61). De esta forma, las investigaciones serían el resultado de un proceso de fabricación, en la medida en que involucran una cadena de decisiones y negociaciones mediante las cuales estas se originan, desarrollan y divulgan.

En segundo término, recogemos los estudios que han destacado el carácter normativo de la actividad científica y el peso que tienen para la constitución de los objetos de estudio las diversas condiciones sociales, económicas, institucionales o políticas que rodean al investigador y a la institución en donde investiga. Los premios, las becas y la orientación editorial de las revistas, las políticas de Estado para el incentivo a la producción científica, la interacción entre investigadores y la competencia entre estos y las características de la institución donde se realiza la investigación, entre otros, son elementos que configuran particulares escenarios que marcan los contenidos y preferencias de los investigadores (Bourdieu, 2003; Íñiguez y Pallí, 2002; Knorr-Cetina, 2005). 
Para estos autores, los intereses particulares producen tácticas de persuasión o dispositivos, culturalmente transmitidos, que influyen en el contenido de la investigación. Sin embargo, dichos contextos solo adquieren significado en relación con la posición que tiene el investigador dentro de dicho escenario y al sentido que él mismo atribuye a esa posición. Así, por ejemplo:

(...) un científico de gran prestigio que dispone de gran crédito en su campo puede permitirse el lujo de tomar decisiones arriesgadas y asumir el fracaso, si es preciso; un científico joven que empieza su carrera y no dispone aún de crédito, puede también asumir el riesgo, puesto que tiene poco que perder (Íñiguez y Pallí, 2002: 19).

Vemos así que la definición del contenido de la investigación estaría influenciada por una posición relativa al contexto y, a su vez, por el sentido dado a la acción de investigar en ese contexto (Bourdieu, 2003).

\section{a) Los procesos de investigación en el campo de la investigación acerca de la formación docente}

En el campo específico que ocupa esta investigación contamos con evidencias y debates en donde se destaca el peso que tienen los contextos, los intereses y la posición del investigador respecto de aquello que se estudia de la formación docente. Diversos autores advierten que las investigaciones en torno a la formación de profesores se orientan hacia problemáticas diferentes dependiendo del rol y la posición de cada investigador, destacando la necesidad de impulsar y valorar las preguntas que emprende el investigadorformador, pues serían estas las de mayor pertinencia para mejorar o transformar los procesos de formación docente (Zeichner, 1998). En una línea similar, Kennedy (1995) destaca en una revisión crítica a las investigaciones realizadas en Estados Unidos que los distintos aspectos de la formación estudiados están relacionados con el ámbito en el que los investigadores desarrollan su labor. Sostiene que hay diferencias claras en los problemas y objetos que se delimitan si, por ejemplo, el investigador se encuentra desarrollando o evaluando políticas de formación docente o si más bien se encuentra vinculado 
al campo como un formador de profesores en algún programa específico.

Otras evidencias las encontramos en la extensa revisión realizada por el panel AERA, donde se analiza la historia de la investigación acerca de la formación de profesores en Estados Unidos desde los años 50, cuando emerge como campo de estudio, hasta nuestros días. Concluyen que la formación de profesores fue estudiada de maneras diferentes, pues el tiempo y el espacio han marcado lo que se consideraba como «prioritario» de ser estudiado. Así, la manera de problematizar la formación en las investigaciones y las aproximaciones metodológicas por las que optan se nutren del contexto histórico y político que rodea a los investigadores (Cochran-Smith y Fries, 2005).

Desde estos parámetros conceptuales suponemos, entonces, que los procesos de investigación sobre la formación docente que se llevan a cabo en Chile, de alguna forma también se guían por estos principios y no escapan a las dimensiones personales, sociales, institucionales, estratégicas y de recursos que es preciso reconocer y analizar por medio de investigaciones empíricas.

2. Diseño metodológico. Investigadores reconstruyendo la génesis de sus estudios

Siguiendo el enfoque teórico asumido, el propósito específico de esta investigación fue reconocer las condiciones personales, contextuales e institucionales que participan en la génesis de las investigaciones en el campo de la formación docente. Dado el carácter exploratorio del estudio, se adopta una aproximación cualitativa y el enfoque de la Teoría Fundamentada. A través de un diseño de estudio de casos se busca comprender el fenómeno por medio del relato de nueve investigadores acerca de los orígenes y antecedentes de sus respectivas investigaciones respecto de la formación docente.

Con un parámetro de «variación máxima de la muestra» (Flick, 2004: 82) primaron en la selección de los participantes del estudio criterios teóricos, de diversidad y disposición del investigador. Por consiguiente, las investigaciones que fueron objeto de análisis aluden a diversos actores, dispositivos y conocimientos profesionales que 
describen o intervienen sobre unos escenarios, procesos y evaluaciones a la formación docente ${ }^{5}$. En segundo lugar se utilizó un criterio temporal: la fecha de finalización de la investigación debía tener un máximo de cuatro años atrás al momento de la entrevista. Esto porque la reconstrucción que hace el investigador acerca de cómo se origina y desarrolla una investigación es más sencilla en estudios terminados recientemente, en comparación con una investigación finalizada hace muchos años. También se cauteló la presencia de estudios diseñados y ejecutados en distintas regiones, con diversidad de financiamientos y representando a variadas instituciones ejecutoras.

De acuerdo con esos criterios, accedieron a participar ${ }^{6}$ en esta investigación nueve investigadores principales, quienes realizaron sus estudios entre los años 2006 y 2008, tal como se detalla en el siguiente cuadro:

Cuadro 1: Caracterización de los investigadores principales considerados para el estudio

\begin{tabular}{|l|l|l|c|}
\hline Participantes & Componentes de la formación estudiados & Tipo de financiamiento & Ubicación \\
\hline Investigador 1 & Dispositivo de formación: prácticas & Interno & Región \\
\hline Investigador 2 & Actor: estudiante de pedagogía y sus conocimientos & Externo (FONIDE) & Región \\
\hline Investigador 3 & Políticas públicas de formación docente & Interno & Centro \\
\hline Investigador 4 & Dispositivos de formación: programas de estudio & Externo (CSE) & Centro \\
\hline Investigador 5 & $\begin{array}{l}\text { Dispositivo de formación: evaluar impacto de } \\
\text { una estrategia }\end{array}$ & Autofinanciado & Región \\
\hline Investigador 6 & Dispositivo de formación: modelo & Externo (FONIDE) & Región \\
\hline Investigador 7 & Actor: estudiante de pedagogía: habilidades básicas & Externo (CSE) & Región \\
\hline Investigador 8 & Dispositivo de formación continua & Externo (FONDECYT) & Centro \\
\hline Investigador 9 & $\begin{array}{l}\text { Dispositivo de formación inicial: foco en } \\
\text { actividades curriculares }\end{array}$ & Externo (FONIDE) & Región \\
\hline
\end{tabular}

Como instrumento principal se utilizó la entrevista episódica (Flick, 2004), la que permitió conocer la historia de la investigación desde su génesis hasta su finalización y contextualizar esos orígenes

5 Un análisis más detallado de esta categorización puede encontrarse en otro artículo publicado en esta misma revista (Cisternas, 2011).

6 Si bien el acceso a los sujetos en cualquier investigación está supeditada a la disposición que estos tengan para participar, en el caso de esta investigación ello resulta particularmente importante, pues suponía que el investigador aceptara convertirse en objeto de investigación. Este cambio de roles y el tema en cuestión para el cual solicitamos la participación (reconstruir detalladamente cómo se originó y materializó su investigación) agregó una dificultad extra, la que finalmente se convirtió en el criterio más importante para definir la muestra. 
dentro del propio itinerario y biografía del investigador, tanto en su acercamiento a la investigación en formación docente, como a su propia experiencia haciendo investigación. Las principales dimensiones que abordó la entrevista fueron las siguientes:

- reconstrucción del proceso de formulación del proyecto de investigación;

- situaciones que marcaron los hitos en el proceso;

- el contexto en el que se situó esta formulación, situaciones que tuvieron injerencia en la definición y el recorte del objeto de estudio; y

- participantes en la elaboración del proyecto, roles y modo en que se configuró el equipo de investigación.

El análisis siguió los principios de la Teoría Fundamentada. Por medio de las estrategias de Conceptualización y Comparación Teórica Constante se avanza desde una codificación abierta a una codificación axial (Glaser, 2002, 2004; Strauss \& Corbin, 2002). Este movimiento se refleja en el proceso de relacionar las categorías y subcategorías emergentes. Se codificó en relación con un eje (una categoría) enlazándolo a los otros según sus propiedades y dimensiones. Estas relaciones dieron cuenta tanto de la estructura como del proceso: Contextos, Condiciones Causales, Condiciones Intervinientes, Acciones e Interacciones y Consecuencias (Strauss \& Corbin, 2002). Para dicho análisis fueron utilizadas dos herramientas específicas: la guía de relaciones condicionales, y la matriz de codificación reflexiva (Scott \& Howell, 2008)

\section{Resultados de investigación: orígenes teóricos y pragmáticos en el proceso de investigar la formación docente}

El análisis revela que la génesis de cada investigación presenta una perspectiva multidimensional. Los factores contextuales e institucionales tienen un rol protagónico en el que se conjugan aspectos de la biografía del investigador. Estas dimensiones están marcadas por la naturaleza del vínculo que tiene cada investigador 
con la formación de profesores. En efecto, la posición del investigador en el campo de la formación docente está fuertemente relacionada con los problemas que delimita y estudia.

A continuación desarrollaremos los principales contextos, escenarios institucionales, disposiciones e intereses individuales y colectivos que los investigadores reconocen como clave en la génesis y desarrollo de sus estudios.

\subsection{La posición del investigador en el campo: investigar para la práctica de formación o para diseñar y evaluar políticas}

Para los investigadores, la cuestión de qué y para qué investigar toma sentido a la hora de explicar cómo ellos llegaron a seleccionar un ámbito de estudio dentro del campo de la formación de profesores. Entre los casos estudiados se distinguen dos opciones bien marcadas: para algunos interesa realizar una investigación in situ, directamente vinculada a los procesos y prácticas de formación, mientras que otros buscan realizar estudios a gran escala, con validez y representatividad nacional, que den cuenta del sistema (o al menos de una parte de él) y que sirvan para el diseño y la evaluación de políticas para la formación docente.

En el primer caso, esto se traduce en un interés por mejorar los procesos, admitiendo que la investigación responde principalmente a la urgencia por resolver problemas prácticos. Habría una condición de estar en la acción que conduce al investigador hacia este tipo de estudios. Dicho interés se presenta cuando los investigadores se relacionan (cotidiana y laboralmente) con el objeto y, en general, coincide con un perfil en donde los investigadores son formadores de profesores, responsables de uno o más cursos de formación y están insertos en Facultades de Educación. También caben aquí los investigadores que comenzaron estudiando la enseñanza, el aprendizaje y las interacciones que ocurren en el aula, o bien, comentan cómo desde allí fueron haciendo un viraje que describen como natural, desde la investigación acerca de la enseñanza hacia la investigación respecto de la formación para la enseñanza. Para estos 
investigadores, además, la docencia y la investigación se enriquecen mutuamente.

(...) yo estaba en la acción, en la sala de clases, en la universidad formando profesores y estaba en la acción tratando de capacitar profesores, me di cuenta de que habia una cantidad de conocimiento que yo misma iba generando (...) entonces por eso que empezamos a trabajar, a transformar este trabajo en investigación, además, porque tú te das cuenta que cuando tú haces eso, tu docencia en la universidad es mucho más rica. (...) O sea, tú empiezas a trabajar en investigación $y$, lo que vas avanzando en investigación, lo vas poniendo en la docencia y lo que vas aprendiendo en la docencia, lo vas poniendo en la investigación, entonces casi tú no te puedes imaginar una docencia sin un trabajo de investigación paralelo. (Caso 8)

Para estos investigadores, la convicción a la que llegan respecto de la necesidad de mejorar o transformar la formación docente surge gracias a su doble condición de investigador-formador, la cual les permite vivenciar u observar incidentes que iluminan e impulsan la delimitación de focos problemáticos específicos. Así, en el origen de estas investigaciones están unos escenarios que desconciertan o frustran al formador, pero que lo iluminan como investigador. El encadenamiento de estos factores (sumado, probablemente, al ejercicio reflexivo de su práctica) retroalimenta, empuja y, en definitiva, se constituye en una condición que facilita la emergencia de investigaciones asociadas al diseño, implementación y evaluación de estrategias y/o herramientas concretas para la formación inicial o continua.

Esta tensión entre lo real y lo necesario de la formación se hace evidente para el investigador-formador básicamente de dos maneras. En primer lugar, cuando experimenta un incidente crítico en la formación, es decir, cuando en escenarios concretos de formación inicial o continua se enfrenta a situaciones desconocidas, poco habituales, que desequilibran sus conocimientos, chocan con sus concepciones, o bien, le impiden dar una respuesta práctica que lo satisfaga. En segundo lugar, esta distancia se hace evidente a los 
ojos del investigador gracias al reclamo y la exigencia explícita que realizan los profesores en formación, es decir, cuando los estudiantes de pedagogía y profesores del sistema que asisten a perfeccionamientos advierten que los contenidos de la formación no se articulan con las necesidades de la escuela y demandan respuestas que sean efectivas para su desempeño profesional. Estos avisos son descritos por un investigador con frustración y desconcierto.

(...) porque los alumnos siempre están como haciéndonos ver las diferencias, tenemos evaluación en las escuelas (centros de práctica), en las diferentes asignaturas y en conversaciones de las diferentes asignaturas los alumnos también se encargan de hacer notar que ellos sentían importante que hubiera una articulación con los diferentes programas o que hubiera una articulación con las prácticas. (...) Y eso pasa también por la autorreflexión primero, no es cierto, de las propias prácticas pedagógicas nuestras. Volviendo al reclamo del estudiante, que son datos empiricos que sirvieron mucho para pensar en esto. (Caso 9)

El reconocimiento de las debilidades que presenta la formación emerge cuando el investigador posee una doble condición profesional. Por una parte, posee vínculos con el sistema escolar, ya que se desempeñó como profesor(a) en establecimientos escolares, asumió algún cargo o actualmente participa en asesorías y formación continua de profesores; y, por otra, es al mismo tiempo un investigador que participa en la formación inicial de profesores. Esta doble condición impulsa al investigador, ya que reconoce las debilidades de la formación debido a que pudo conocer el sistema escolar por dentro. Es en su posición de investigador-formador en donde comprendemos su interés por la investigación en este escenario.

(...) yo estaba absolutamente metida en la formación inicial, o sea, yo toda mi vida trabajé en formación inicial, y yo me daba cuenta de que lo que se estaba haciendo en formación inicial no era exactamente lo que se necesitaba para el aula, porque yo trabajaba en el fondo con profesores de aula, porque iba a trabajar, en los estudios que yo estaba haciendo, trabajaba con ellos, al mismo tiempo trabajaba formando profesores en la universidad, yo me daba cuenta 
que habia como una... una distancia... yo no quiero decir que pensaba que estaba todo malo lo que estábamos haciendo, porque yo era parte integral de lo que estábamos haciendo. (Caso 8)

En suma, este grupo de investigadores reconoce como crucial en el proceso de delimitar sus intereses investigativos la convicción a la que llegan respecto de la necesidad de mejorar o transformar la formación docente, misma que surge gracias a su doble condición de investigador-formador que le permite vivir conflictos que lo iluminan para darse cuenta y visualizar allí un nicho de investigación.

Por otra parte, encontramos un segundo grupo de investigadores cuyo interés se sitúa en el ámbito de las políticas y decisiones institucionales para la formación docente. Observamos que en estos casos los propósitos de la investigación cambian: el foco está en las investigaciones a mayor escala (ya no centradas en un programa específico) y que sirven para evaluar, mejorar y/o diseñar políticas de formación inicial y continua. Formulan así estudios que buscan evaluar el impacto a gran escala de una política implementada, recogen información de poblaciones significativas como para dar cuenta de tendencias en el sistema de formación, o bien cuando menos, buscan la manera de comparar aspectos significativos entre varios programas formativos.

¿Quiénes son estos investigadores? A diferencia del investigadorformador se trata de personas e instituciones que de alguna manera han estado vinculadas con el diseño o evaluación de políticas y programas específicos orientados a mejorar la calidad de la formación docente en el país. Sus razonamientos se justifican en la necesidad de tener datos duros para convencer, denunciar y visibilizar temas de interés nacional. A juicio de ellos, esos temas son parte de la agenda pública y por eso se discuten ampliamente, pero dichas discusiones a menudo carecen de investigaciones que las sustenten. Además, reconocen el rol protagónico que debe cumplir este tipo de investigación cuando se requiere conocer el impacto de una política o programa.

En síntesis, son dos escenarios y posiciones diferentes que anidan investigaciones de distinta naturaleza. Con el primer grupo, 
el objeto se construye a nivel micro, es decir, en relación con las características y procesos de un programa particular, que se traduce en estudios que sirven principalmente al formador de profesores y a los programas de formación. A su vez, en el segundo grupo se construye un objeto a nivel macro, es decir, con estudios de mayor escala que buscan comparar varios programas, sus características, o bien, el impacto que alcanzan entre los profesores en formación, arrojando resultados que pueden servir menos a los formadores y mucho más a los decisores, asesores, diseñadores de políticas a nivel regional y nacional o evaluadores de estas.

Figura 1: Posición del investigador en el campo de la formación docente

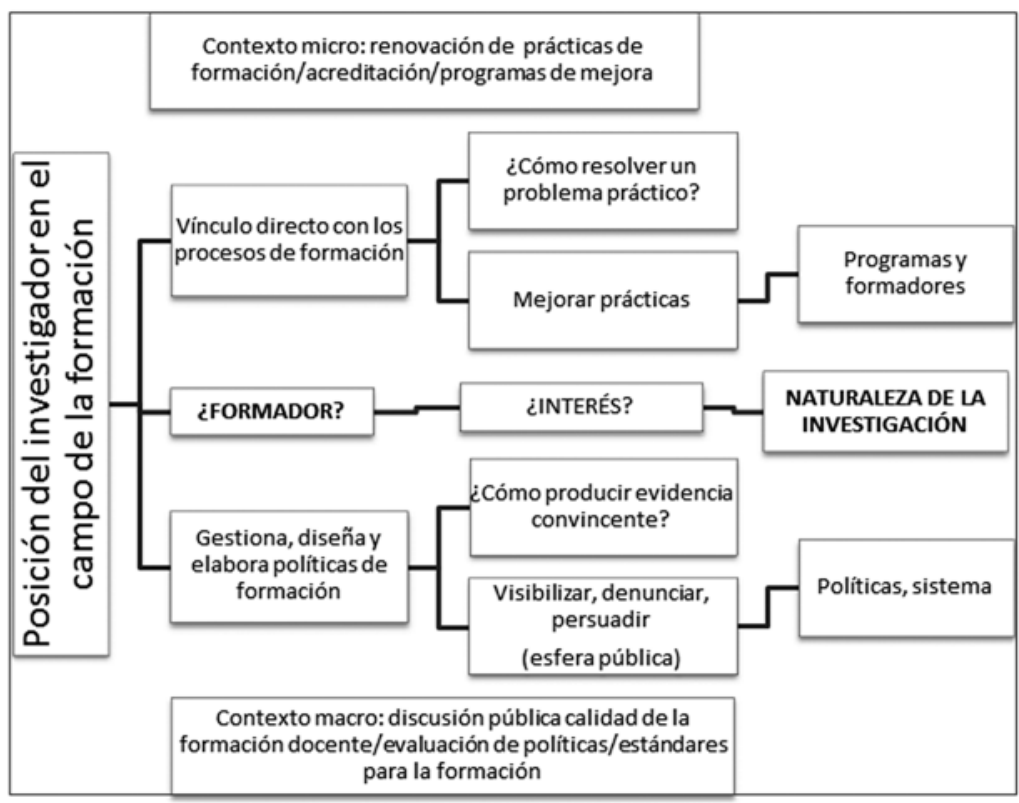

3.2. Estandarización del oficio de investigar: hay que cumplir ciertos requisitos para entrar al club

Otra dimensión que se levanta en torno al origen de una investigación es el definido por las convocatorias a concurso para acceder a fondos de investigación. Resulta sorprendente la coincidencia entre los investigadores que estudian la formación inicial y continua cuando explican cómo se sintieron interpelados cuando se oficializó un determinado llamado a concurso. Tal parece ser que estas instancias 
se convierten en una especie de chispa que desencadena una serie de decisiones en torno a la investigación.

Estas situaciones, de naturaleza contextual y contingente, ejercen una presión significativa no solo en términos de los recursos para materializar un estudio, sino también como dispositivos que movilizan la problematización y el recorte de lo investigado en este terreno.

Sin embargo, no basta con el impulso y la decisión de postular. Un segundo paso supone formular un proyecto que cumpla con los estándares exigidos por la comunidad de investigadores y las instituciones financistas. Llama la atención la mención reiterada y transversal entre los casos respecto del proceso mismo de formular la investigación como una instancia gravitante de la definición del objeto a estudiar. Ilustran de qué forma no poseer ciertos antecedentes puede llegar a limitar la posibilidad de concursar con éxito y, en consecuencia, investigar acerca de sus temas específicos de interés. Este conjunto de exigencias y requisitos supone que los investigadores demuestren estar en posesión de suficientes antecedentes que reflejen un posicionamiento y consolidación en el contexto de la investigación en educación.

El análisis muestra un fenómeno interesante en este punto. Varios de los participantes relatan que no cumplían con algunas de estas exigencias y, sin embargo, pudieron acceder igualmente a los fondos gracias al desarrollo de unas estrategias o tácticas que, a juicio de ellos, permitieron ganar concursos y concretar sus ideas. Las condiciones y estrategias más importantes para estos investigadores fueron: acreditar una posición como investigadores, actualizar el conocimiento teórico y empírico del objeto a investigar y buscar apoyo en terceras personas que posean experiencia y bagaje en concursos de prestigio. Veremos cada una de estas estrategias por separado.

a) Acreditar una posición de investigador con trayectoria

A partir del análisis y desde el propio discurso de los investigadores se produce una especie de círculo virtuoso con aquellas investigaciones en torno a la formación docente que logran obtener acceso a fondos 
prestigiosos. Esto, porque trae una serie de beneficios para el investigador, su equipo y la propia investigación.

Ganar un fondo de financiamiento competitivo y de prestigio, señalan, abre la puerta para desarrollar una línea de investigación, permitiendo que los equipos se constituyan y consoliden. Facilita la divulgación y circulación de los resultados, lo que permite iniciar nuevamente este círculo virtuoso, acumulando prestigio entre la comunidad de investigadores y dando acceso a fondos para seguir investigando. Pero lo que se suele ignorar es que acceder a estos beneficios implica que los investigadores puedan acreditar una posición. Veamos cómo explican esta condición dos investigadoras: la primera, con una experiencia poco exitosa, pues aún no ha podido acceder a financiamientos de mayor envergadura, pese a haber postulado en varias ocasiones y, la segunda, una investigadora que ha ganado sucesivamente varios concursos y tiene un alto prestigio, sin embargo, relata lo difícil que fue entrar al club.

¡Claro que he postulado! He postulado al fondo XXX, pero nunca se han ganado los proyectos, yo creo que tiene que ver un poco con que yo no tenga el grado, esa creo que es una de las explicaciones. La otra es que yo si bien tengo bastantes publicaciones, esas publicaciones no son las validadas por las investigaciones y si tú ves cómo este fondo nace, se originó como politica justamente para académicos como nosotros. Pero si tú miras su formulario de postulación al final terminó siendo igual de exigente que otros fondos, (...) pero hoy día te agregaron como exigencia que coloques todas tus publicaciones SCIELO e ISI. (Caso 5)

Fue difícil al principio, para meterse en el rodaje, o en el club del fondo XXX -iporque es como eso! (risas)-. Yo no tenía grados académicos, porque yo soy súper vieja (...) yo decía por qué le voy a restar tiempo a la crianza de mis hijos, para ir a hacer un magister de administración en educación, una cosa que no era lo mío, pero que en la universidad me decian "pero XXX, es súper importante que tú hagas, no sé qué y no sé qué...» y yo siempre me negué a eso, pero al final de mi carrera eso me empezó a pesar, porque quedé fuera del club, ¿te fijas?, pero a pesar de que quedé fuera del 
club de investigadores, con grado y con título, yo presentaba proyectos y me los ganaba (risas). Y los otros me miraban como diciendo (...). En el fondo me respetaban solo porque me ganaba los proyectos, de lo contrario, no me hubiesen respetado jamás, ¿te fijas? Y al final hice historia, hay caminos que son distintos a los tradicionales y que pueden llegar a las mismas metas. (Caso 8)

Como vemos, para los investigadores el criterio de calidad implica al menos tres requisitos: poseer un grado académico, contar con experiencia previa en investigación y publicaciones en revistas indexadas. Cumpliendo con estos criterios se facilita el acceso a recursos y, por lo tanto, a materializar sus propias ideas de investigación.

Dentro de los investigadores participantes en el estudio distinguimos dos grupos: para unos, acreditar estas condiciones se presentaba como un obstáculo, mientras que para otros no era más que un trámite de poca relevancia dentro de la génesis de la investigación. Esto último ocurre cuando se trata de un investigador que ya posee esos antecedentes y los entiende como parte de las reglas del juego, a diferencia de aquellos que investigan por primera vez y desconocen o no comparten estas condiciones.

b) Utilizando y recontextualizando aportes actualizados de otras investigaciones

El análisis reflejó que las investigaciones con financiamiento de prestigio a nivel nacional tienen un rasgo común: en el origen y delimitación de sus objetos de investigación fue necesario situar sus intereses dentro del debate teórico y empírico que otros investigadores ya habían realizado dentro del mismo objeto de atención, es decir, debieron reconocer y dialogar con otras investigaciones similares, buscando puntos en común y espacios de diferencia para enmarcarse en las discusiones teóricas y empíricas propias del objeto. Esta estrategia es clave e inherente al oficio de investigar y se la reconoce como central al momento de definir la relevancia de una investigación. 
Para actualizarse teórica y empíricamente respecto del objeto que se está estudiando, estos investigadores saben que al mirar el desarrollo de las líneas de investigación se pueden recoger nuevas preguntas que otros van formulando. Se trata de seguir los caminos realizados por quienes investigan problemas similares, usándolos para recontextualizarlos, desandarlos, o bien, proponer un nuevo camino que sea acorde con los contextos e intereses que motivan al investigador. Para lograr esto, describen una serie de decisiones que acompañan al proceso de delimitación del objeto de investigación, por ejemplo: uso de resultados de investigaciones propias, diálogo con investigadores de prestigio, análisis de otros estudios acerca del objeto, adaptación a la idiosincrasia nacional de los conocimientos elaborados en otros contextos o de contextos particulares.

Constatamos que el diálogo y la colaboración con otros investigadores son factores decisivos en la delimitación de los objetos investigados, especialmente cuando establecen vínculos académicos a nivel internacional.

[La investigadora responde a la pregunta: ¿Por qué quisieron mirar aquello?, ¿qué discusiones, si recuerda algo, cómo fueron llegando a delimitar ese objeto?] Es que no éramos nosotros los únicos que estábamos discutiendo eso, o sea, casi todos los grupos con los cuales nos relacionábamos estábamos en las mismas. (...) Es súper curioso cómo el camino que va siguiendo la investigación de un área del conocimiento es similar en grupos que no se han visto nunca. Entonces, después tú te empiezas a juntar y te das cuenta por qué razón, primero todos quieren ver cómo se enseña y después cómo se evalúa. (...) Todos partimos viendo cómo funcionaba el conflicto cognitivo y después nos dimos cuenta de que el conflicto cognitivo no funcionaba siempre. (Caso 8)

El análisis nos permitió explorar también las condiciones y contextos que subyacen en el uso de estas estrategias como fundamentales para el origen de una investigación. Desde una perspectiva más normativa sobre lo que debiesen ser las prácticas de investigación, podríamos señalar que circunscribir, delimitar y formular una investigación a partir de los distintos enfoques 
conceptuales y metodológicos es un requisito mínimo para cualquier estudio. A priori, creíamos que los investigadores destacarían estas prácticas como fundamentales para explicar el origen específico de una investigación. Más aún, esperábamos poder encontrar en los discursos de los investigadores distintos discursos teóricos acerca de la formación que había servido de escenario para sus opciones y formulaciones. Sin embargo, esta dimensión no se presentó en la génesis de todas las investigaciones. El análisis de los datos permitió observar que la revisión de otros estudios y el diálogo con las propuestas de otros investigadores se materializa bajo circunstancias bien particulares.

En primer término, el uso de lo que otros han investigado es una práctica presente entre quienes han desarrollado estudios previos en el campo y vinculados al mismo objeto, es decir, cuando poseen cierta trayectoria o permanencia en el estudio de temáticas y problemas similares. En segundo lugar, dicho uso se observa cuando el investigador tiene una formación previa especializada en lo que será su objeto de estudio, lo cual le entrega un amplio manejo teórico acerca de este y le permite insertarse más fácilmente en un campo específico y sus desarrollos. Al contrario, cuando se trata de investigaciones que por primera vez realiza el investigador, o bien, donde este declara estar «empezando» a estudiar un área, pues le parece altamente relevante, este diálogo teórico y consideración del conocimiento disponible no tiene presencia como una dimensión gravitante en la génesis de un estudio. Nuevamente surge como factor explicativo la distinción entre investigador experimentado e investigador principiante.

c) Buscando apoyos sin riesgos: quien a buen árbol se arrima, buena sombra lo cobija

A partir de los casos estudiados observamos que las investigaciones se materializan muchas veces gracias a tácticas que utilizan los propios investigadores para asegurar la calidad de la postulación y, con ello, aumentar su competitividad y posibilidades de ser elegida. Ya mencionamos dos de esas estrategias: acreditar una posición y usar lo que otros han investigado y recontextualizarlo. Una tercera vía comúnmente utilizada para concretar (y a veces delimitar) una idea 
de investigación es la búsqueda de apoyos en investigadores con un mayor bagaje y experiencia exitosa en concursos. Al parecer, habría un conocimiento específico, difícil de transmitir formalmente, que se alcanza solo gracias a la vivencia específica de diseñar, concursar y ganar un proyecto de investigación. La racionalidad que subyace es que a mayor cantidad de concursos ganados, mayor es el conocimiento respecto de cómo hacerlo. La siguiente cita ilustra el funcionamiento de esta táctica.

I: Entonces, ya tenía la inquietud de que algo estaba pasando, ahora, fue de un rato para otro cuando digo "ibaja los formularios del fondo XXX!» (ríe) Y nos apoyamos de XXX que ya tenía más experiencia en proyectos de este tipo. Ella nos dijo: aquí vamos con los objetivos y empezamos a formular los objetivos.

\section{E: ¿Quiénes participaron ahí?}

\section{I: Participó fuertemente XXX.}

E: ¿Por qué ella?

I: Ella porque ella es la directora del posgrado aquí y tenía antecedentes de haber ganado proyectos antes ite fijas? Entonces ella tenía experiencia en cómo se comunican tus ideas de investigación en un proyecto concursable. (Caso 2)

Una variación en la estrategia, que sin embargo actúa bajo el mismo principio, es incorporar en el equipo, de forma estratégica, a investigadores destacados o con reconocimiento en el medio. En estos casos, interpretamos que la trayectoria y experiencia del investigador que se incorpora al equipo finalmente se transfiere a la propuesta investigativa, dándole credibilidad y dejando en un segundo plano las posibles «debilidades» del resto del equipo, como por ejemplo, que alguno de los participantes no tenga certificados o trayectoria suficientes para cumplir con las exigencias del concurso.

Contrario a una hipótesis que barajábamos, en estos casos los investigadores experimentados, en general, no intervienen respecto de qué se investiga, es decir, no impactan conceptual o 
metodológicamente sobre el objeto de la formación estudiado. Los investigadores que recurren a dichas estrategias conservan cierta autonomía de la idea original de investigación y, al menos en los casos analizados, no hay indicios de que los investigadores que acompañan con su nombre el proyecto hayan modificado el problema que originalmente quería abordar ese equipo. La siguiente cita refleja la incorporación estratégica de un investigador prestigioso en el equipo:

(...) Porque tú sabes que para postular a una investigación hay que tener trayectoria técnica. Entonces ella se ofreció a participar en este grupo y avalar con su propia investigación las potencialidades nuestras, digamos. Nosotras teníamos muy poca productividad.

E: ¿Y cómo se integra la cuarta investigadora?

I2: Era una condición estratégica (risas).

11: Bueno, porque nosotras si mirábamos nuestra productividad no teníamos mayores cosas en la educación. Excepto lo que tenía yo, que era una investigación interna de la universidad, con un par de cosas, pero no teníamos análisis (estudios) importantes. (Más bien) nada que mostrar, o nada que se conociera en el ambiente, entonces, esto también para nosotros fue un aval importante. (Caso 9)

Detrás de estos procesos vemos que simultáneamente conviven gestos de confianza e interés. Cuando se integra un investigador de prestigio a un proyecto, este gana un antecedente más que fortalece su posición destacada en el medio. Sin embargo, no se trata de entregar a ciegas el capital simbólico que trae consigo este investigador experimentado. El investigador de prestigio debe creer en el proyecto al cual se unirá y debe estar seguro de las competencias investigativas del resto del equipo. Es decir, no se arriesga si no hay confianzas de base. Nuestras investigadoras nos lo hacen ver muy claramente:

[Refiriéndose a la investigadora de prestigio que participó en la investigación] Yo creo que también ella sentía que no se estaba exponiendo. Parece que también está el ego docente y en la medida en que las personas van adquiriendo cierto 
prestigio, las personas cuidan eso. (...) Esto también para nosotros fue un aval importante, pues significaba que creía en nosotras. (Caso 9)

¿Por qué recurrir a estas tácticas? Se admite la necesidad de manejar un lenguaje específico (lenguaje de concurso), el cual se alcanza más fácilmente cuando hay una experticia acumulada después de haber postulado y ganado proyectos. En segundo lugar, los postulantes saben que estos investigadores de prestigio han actuado también como evaluadores de proyectos, es decir, conocen el medio y sus criterios. Por lo tanto, en la definición de un proyecto de investigación pueden ofrecer esta información. En tercer lugar, también existe un criterio asociado a las características del objeto estudiado: si el propósito es visibilizar o tener un impacto en las políticas y en el debate público, entonces la estrategia de incluir a un investigador prestigioso en el equipo se hace mucho más relevante.

A XXX [se refiere a la investigadora] era obvio que habia que invitarla [se entiende que por su prestigio, pues señala a una investigadora ampliamente conocida en el medio]. Y a XXX, aunque tenía otra agencia, era conocida de XXX y de XXX. Y yo no la conocía mucho, pero sí sabía de ella. Entonces era una persona obvio que habia que invitar, por los conocimientos y por su nombre, o sea, siendo bien franco (...) también nos importaba el nombre digamos y que estuviera ella ahí. (Caso 4)

En suma, habría un contexto que propende a la estandarización en la medida en que hay requisitos homogéneos y, a la vez, dicho contexto alienta la búsqueda de estrategias para poder ser incluido en ese rodaje o club. Los formularios de postulación se presentan como un artefacto mediante el cual se debe volcar una idea de investigación, un objeto de estudio y además demostrar estar en posesión de antecedentes que reflejen una historia de productividad investigativa con estándares científicos. Todo lo anterior deja ver el carácter oportunista que tiene el ejercicio de la investigación en este campo y la importancia de mantener una actitud de resistencia, pero también perseverante y estratégica en escenarios exigentes y competitivos. 


\subsection{El peso de la institución ejecutora: centralismo, trayectoria y presiones}

Este aspecto se caracteriza por ser altamente contextualizado, ya que se centra en escenarios que pueden facilitar u obstaculizar el origen de una investigación en el campo de la formación docente. En este sentido, la dificultad inherente al proceso de formular y concretar un estudio dependerá de algunos rasgos de la institución, como pueden ser su ubicación (esto es, del centro, o bien, de una región), su nivel de desarrollo y el grado de valoración que esta otorgue a la actividad investigativa y las presiones que esta misma institución ejerce para impulsar la actividad investigativa entre los formadores. El siguiente cuadro refleja el resultado del análisis. Posteriormente daremos cuenta de estos aspectos por separado.

Cuadro 2: Matriz condicional: Las «huellas» de la institución ejecutora (donde se anidan los investigadores)

\begin{tabular}{|c|c|c|c|c|c|}
\hline Qué & Cuándo & Dónde & Por qué & Cómo & Consecuencia \\
\hline $\begin{array}{l}\text { Ubicación } \\
\text { de la } \\
\text { institución } \\
\text { facilita o } \\
\text { restringe }\end{array}$ & $\begin{array}{l}\text { Ser de región } \\
\text { v/s del centro } \\
\text { (Santiago) }\end{array}$ & \begin{tabular}{|l|} 
Llamados \\
a concurso \\
para obtener \\
financiamiento
\end{tabular} & $\begin{array}{l}\text { Los debates y } \\
\text { comunidades están } \\
\text { en el centro } \\
\text { Financiamiento } \\
\text { excluye ISI- } \\
\text { SCIELO (en región } \\
\text { hay menos) } \\
\text { Investigadores con } \\
\text { créditos y prestigio } \\
\text { están en Santiago }\end{array}$ & \begin{tabular}{|l} 
Trato desigual \\
Distintas \\
condiciones \\
Financiamiento: \\
ser de región \\
hace más difícil el \\
acceso \\
Evaluadores \\
son también \\
investigadores
\end{tabular} & $\begin{array}{l}\text { Ser de región } \\
\text { demanda más } \\
\text { esfuerzos para } \\
\text { investigar }\end{array}$ \\
\hline $\begin{array}{l}\text { Desarrollo y } \\
\text { valoración } \\
\text { de la } \\
\text { actividad } \\
\text { investigativa } \\
\text { facilita o } \\
\text { restringe }\end{array}$ & \begin{tabular}{|l} 
Mayor o \\
menor \\
trayectoria \\
de la \\
institución
\end{tabular} & $\begin{array}{l}\text { Universidad } \\
\text { con historia } \\
\text { Universidad } \\
\text { en sus inicios }\end{array}$ & $\begin{array}{l}\text { Prestigio de la } \\
\text { universidad } \\
\text { avala/condiciona } \\
\text { financiamiento } \\
\text { Investigar es un } \\
\text { oficio: demanda } \\
\text { tiempo } \\
\text { Sin financiamiento, } \\
\text { no hay formación } \\
\text { de equipos }\end{array}$ & \begin{tabular}{|l|} 
Institución \\
presiona para \\
postular \\
Formación \\
de equipos: \\
institución \\
presiona para su \\
configuración \\
Universidad \\
sin interés por \\
investigar \\
\end{tabular} & $\begin{array}{l}\text { Investigación } \\
\text { independiente } \\
\text { no tiene } \\
\text { reconocimiento } \\
\text { Sin apoyo } \\
\text { (independiente) } \\
\text { obliga a buscar } \\
\text { recursos }\end{array}$ \\
\hline
\end{tabular}

a) Centralismo: es difícil ser de región

Una de las condiciones que los investigadores mencionan recurrentemente es la desventaja que se produce entre una universidad 
de región y una del centro (Santiago). Quizá la fuerza de esta dimensión está marcada por el número mayoritario de casos que provienen de región, sin embargo, los investigadores que están en el centro coincidieron con el diagnóstico.

Esa brecha se refleja principalmente en las condiciones, siempre diferentes, bajo las cuales se investiga en uno y otro lugar. En este sentido, ser de región demandaría mayores esfuerzos, porque los debates y las comunidades se ubican, generalmente, en el centro. Los investigadores destacan que el financiamiento casi siempre excluye a quienes carecen de publicaciones indexadas. Este último factor tiene un fuerte efecto, ya que en las regiones hay menos posibilidades de alcanzar estos estándares debido a que los investigadores con créditos y prestigio se concentran en la Región Metropolitana, donde estos recursos están igualmente concentrados.

Sin embargo, esa misma condición también es asumida como una oportunidad para focalizarse en los verdaderos problemas y necesidades del campo, más que en seguir modas o tendencias de lo que se esté haciendo en el centro. En cierta forma, interpretamos que ser de región daría mayor libertad e independencia en la formulación de problemas de investigación.

Una investigación que no sea en el centro, siempre está constituida como un desafío, yo creo que para todos en general es un desafí que se le suman estos temas de contextos, pero de contexto propio local, pero también de contexto emocional, en que nos están midiendo con el de Santiago, o con el de las grandes ciudades, o con universidades que supuestamente tienen mayor prestigio (...) y eso es una permanente prueba, yo creo que eso es un aspecto que no se puede separar, permanente desafio de ir mostrando que soy capaz, estar mostrando que además los estudios que se realizan sean un verdadero aporte. (Caso 7)

Tal como ocurre con la trayectoria del investigador, esta también puede ser una condición clave en lo que respecta a asumir o no el riesgo de abordar preguntas novedosas, o bien, utilizar enfoques teóricos y metodológicos originales. 
b) Institución ejecutora: trayectoria y prestigio que avalan

Tal como lo vimos con el investigador de elite, el prestigio de la institución también se traspasa tanto al investigador como a su propuesta de investigación. En estos casos, las ideas de los investigadores tuvieron mayor poder de convicción cuando estaban ancladas en una institución que daba garantías con su trayectoria y prestigio. Y, a la inversa, cuando la institución carecía de estas cualidades, el investigador debió aumentar sus esfuerzos para conseguir recursos, buscar apoyos estratégicos o ejecutar su investigación de forma independiente o con apoyos voluntarios. Este hallazgo se observa de manera transversal en los casos analizados.

Este fenómeno fue clave para el origen de algunas investigaciones que hacían innovaciones en el estudio dentro del campo de la formación docente. El prestigio de la institución permite que una investigación para la práctica realizada por un investigador-formador tenga más posibilidades de convertirse en investigación de elite. Esto resulta llamativo, pues como lo señalamos en el primer punto de estos resultados, se asocia la investigación de mayor prestigio a aquella de gran escala, que analiza y propone aportes a las políticas nacionales. Sin embargo, el análisis da cuenta de indicios en donde la doble condición de investigador de elite y formador-investigador coincide con una investigación que puede catalogarse como de prestigio, pues el investigador participa de un equipo que ha ganado concursos de alta importancia sistemáticamente en los últimos 15 años. ¿Cómo lo logró?, ¿por qué en este caso no existe esa aparente incompatibilidad? La propia investigadora nos entrega una pista de su éxito:

[La investigadora responde a la pregunta: ¿cree que con las mismas ideas pero en otra institución, quizás con menos prestigio, habría tenido el mismo respaldo financiero, el respaldo institucional, la atención de la comunidad para realizar esta investigación?

No, yo creo que no, yo creo que en ese momento no, en esa época la universidad XXX tenía un prestigio muy alto y eso te avalaba, obviamente. Si va una persona de «perico los palotes», a postular al fondo XXX, solo si hay antecedentes las comisiones evaluadoras lo consideran, obviamente, de lo 
contrario piensas que no hay respaldo atrás de esa persona, entonces yo creo que es poco probable que nuestras ideas hubieran florecido. (Caso 8)

Esto nos hace pensar, entonces, que la investigación que sirve a los formadores y más directamente a los programas de formación se convierte en investigación de elite cuando la institución ejecutora, donde se anida el investigador, tiene un prestigio que la hace sobresalir entre las otras instituciones y, por tanto, le transfiere a esa investigación y al investigador su credibilidad frente a la comunidad. Más aún, podríamos decir que un organismo financista (que también tiene sus propios intereses y visiones acerca del estudio en el campo educativo) incluso cuando no coincida o no propenda al impulso de ciertos ámbitos o temas de investigación, se arriesga y se la juega por financiarlo, porque se siente avalado por el prestigio y la trayectoria de la institución que apoya a ese investigador.

¿Qué hace que una institución sea portadora de esta reputación? Aunque el estudio no explora el significado concreto que supone tener prestigio institucional, observamos que las instituciones donde se anidaron los estudios que estamos analizando reúnen dos factores que interactúan entre sí. Primero, los recursos disponibles para investigar sumados a la presencia de equipos e investigadores de prestigio entre sus filas y, en segundo término, una historia que distingue a esa institución respecto de otras por su sostenido y sistemático acceso a recursos competitivos en investigación. Vemos así que las universidades con años de trayectoria en investigación educativa se diferencian de aquellas universidades que se inician y funcionan de manera más precaria o que, pese a contar con recursos, no muestran una política clara de apoyo a la investigación en este campo.

Así mismo, a diferencia de una institución sin antecedentes en investigación, las que ya han ganado trayectoria y crédito utilizan estrategias de incentivo y presión para que los investigadores constituyan equipos y postulen a financiamiento.

Bueno, la verdad es que lo «tiramos» porque el decano nos estaba presionando a varios grupos para que hiciéramos la 
postulación al fondo XXX y bueno dijimos, si gana, gana y si no, bueno también, no se nos iba la vida ite fijas? (Caso 2)

El análisis refleja que las huellas de la institución respecto del desarrollo de la investigación en este campo pueden ser de naturaleza directa e indirecta. Diremos que son directas cuando la institución formula políticas propias de incentivo a la investigación y, en particular, a la postulación a concursos; e indirectas, cuando la institución influye por «su nombre» (es decir, su posición en relación con las otras instituciones y el capital que ha acumulado) en la valoración que se hará de un proyecto antes de ser ejecutado, o bien, frente a una investigación terminada.

\section{Conclusiones: las arenas transepistémicas en el proceso de investigar la formación docente}

La relación del investigador con el campo (de la práctica o el de las políticas) junto con sus intereses por investigar en niveles micro o macro, son antecedentes importantes cuando opta por unos focos de interés y renuncia a otros. Ello determina que las preocupaciones del investigador estén marcadas por la posición que este ocupe en el campo. Así mismo, las postulaciones al financiamiento de la investigación se convierten en sí mismas en una especie de artefacto que tiene consecuencias no solo en el origen, sino también en el recorte de los problemas que abordan las investigaciones. A partir de los hallazgos y a modo de conclusión, destacaremos tres ideas.

La inmadurez y precariedad de la investigación en el campo

A lo largo del relato acerca de las dimensiones que participan en la definición de las investigaciones, hemos recogido una serie de evidencias de distinta naturaleza que reflejan la inmadurez del campo de la investigación respecto de la formación docente desde el punto de vista de las condiciones en las que esta se desarrolla. Esta inmadurez la vemos expresada en el protagonismo que alcanza la contingencia situacional (Knorr-Cetina, 2005), la que actúa empujando más de lo que quisiéramos el estudio de la formación docente. 
En efecto, el análisis reflejó que la definición de unos objetos de estudio solo a veces se ancla en discusiones actualizadas y la revisión del estado de la investigación sobre el objeto de estudio. Más bien, estas prácticas se presentan de manera secundaria, reducidas o descartadas frente a otras situaciones que asumen mayor protagonismo en la génesis de los estudios, como por ejemplo, los incidentes específicos vividos por el investigador-formador, el llamado a concurso que impulsa a pensar y a seleccionar un foco, o bien, a la invitación (presión) que hace la propia institución en donde se encuentra el investigador y que impulsa a buscar y delimitar un foco.

Otra evidencia en favor de este argumento proviene de las estrategias que precisan ser usadas por los investigadores para ejercer la investigación con recursos suficientes: la necesidad de aprender a usar un lenguaje de proyectos e incluir a investigadores prestigiosos que avalen sus ideas es una muestra de la inmadurez de este campo.

En una perspectiva más amplia, podríamos preguntarnos si estas son características exclusivas de la investigación respecto de la formación docente o si más bien esta condición de desarrollo representa en realidad a todo el campo de la investigación educacional en nuestro país. Aunque no tenemos antecedentes suficientes para responder a esta interrogante, coincidimos con algunos autores (Brunner y Salazar, 2009; CIDE y MINEDUC, 2007; Tenti, 2001) acerca de la precariedad de los procesos de producción de conocimiento en el ámbito educativo.

\section{Las arenas transepistémicas en la investigación en torno a la formación docente}

¿Por qué, mientras esperábamos hablar con los investigadores respecto de sus objetos de estudio y enfoques de la formación docente, terminamos escuchando mucho más acerca del financiamiento, las postulaciones, las estrategias de conformación de equipos o el papel de la institución ejecutora? En parte, ya lo dijimos en el punto anterior, esto se entiende por la inmadurez de este espacio investigativo. Sin embargo, ello es cierto solo en parte. También se explica por la perspectiva teórica del estudio: los productos de la investigación 
llevan las marcas del contexto en el que fueron producidos y esta contextualidad va más allá del propio objeto de investigación y más allá del propio ejercicio investigativo (Knorr-Cetina, 2005). Diversos autores han puesto en duda que la comunidad de pares investigadores y el estado de conocimiento alcanzado en sí mismos expliquen cómo se levantan y relevan unos temas y problemas (Bourdieu, 2003; KnorrCetina, 2005; Latour y Woolgar, 1995). En este sentido, los hallazgos reconocen las condiciones contextuales, biográficas e institucionales implicadas en la definición de aquello que se estudia en un campo específico.

Si atendemos a los resultados de esta investigación, la urgencia por validar el diseño de un dispositivo de formación para aplicarlo en una facultad de educación, la decisión de agregar un componente metodológico cuantitativo para convencer al organismo financista, esperar el financiamiento de una institución prestigiosa para poder ejecutar la investigación con respaldo o la necesidad de reunir a investigadores que trabajan por separado para hacer una alianza para futuros proyectos son indicios de la influencia de otros ámbitos que no se relacionan con el objeto de la formación docente. Esas son las arenas transepistémicas de la investigación en torno a la formación docente, pues nos remite a redes de relaciones simbólicas que en principio van más allá de los límites de una comunidad de investigadores o del campo específico de investigación y cuyo componente principal está dado por unas relaciones de recursos (Knorr-Cetina, 2005).

\section{Posición del investigador: la fuerza de «quién lo dice»}

Otro elemento a destacar en las condiciones de emergencia de las investigaciones revisadas, es el relativo a las características de quién investiga y qué es lo que ese sujeto investiga. Vemos ciertas características que poseen algunos investigadores y que facilitan el acceso a recursos y, consecuentemente, hay algo de lo que carecen otros investigadores y que limita sus posibilidades de hacer investigación. En este sentido, Bourdieu sostiene que «el capital simbólico va al capital simbólico: el campo científico da crédito a los que ya lo tienen» (Bourdieu, 2003: 102). Es decir, son los investigadores más conocidos quienes aprovechan la mayoría de los beneficios simbólicos 
aparentemente distribuidos en partes iguales. Por ello la importancia que tiene la posición del investigador, la que está configurada por su trayectoria personal y por la institución que lo cobija.

Desde este punto de vista, comprendemos algunas estrategias usadas por los investigadores. Constatamos que, por ejemplo, buscan apoyo en un investigador con trayectoria para que participe de un proyecto, o bien, a un investigador cuyo nombre le agregue mayor credibilidad e impacto a los futuros resultados de la investigación. En esas estrategias destaca la importancia que tiene el propio investigador (con su capital) para validar preguntas y resultados de investigación en el área.

Sin embargo, esta condición también puede llevar consigo algunas consecuencias menos deseadas: si solo aquellos investigadores que poseen determinadas características y posición están autorizados para formular y desarrollar el conocimiento en el campo de la formación de profesores, ¿cómo garantizar que sean ellos quienes desarrollen la investigación en la dirección que la formación docente de nuestro país necesita?, ¿y si esas propuestas provienen de investigadores con menos reconocimiento y apoyo institucional? Creemos que la posición del investigador puede ser un rasgo, pero no garantiza la pertinencia de la investigación para los desafíos que enfrenta el país. Por ello, atendiendo a la condición emergente de este campo en Chile, sugerimos que los caminos que siga la investigación respecto de la formación docente pueda trazarse asegurando que la pertinencia y la calidad de una propuesta investigativa tenga mayor peso que la posición de quien la impulsa.

\section{La importancia de desnaturalizar el proceso de investigar}

En definitiva, el estudio que hemos reseñado entrega evidencias que permiten resituar al investigador como actor en contexto, con historia, posición y opciones frente a la educación, la formación y la investigación. Además, aportamos evidencia para comprender las prácticas de investigación más allá de una actividad exclusivamente racional. Ella también implica creatividad, estrategias, decisiones, sufrimientos e ilusiones. 
Observamos de qué manera las decisiones respecto de qué y cómo investigar nunca resultan ser neutras, ni están predefinidas o guiadas por unas reglas estrictas, estáticas y uniformes. Al contrario, existe una amplia gama de condiciones que intervienen de distinta manera en este proceso y estamos convencidos de que este tipo de análisis provee de herramientas para alejar perspectivas ingenuas acerca de la producción de conocimiento en el campo educativo.

\section{Referencias}

Brunner, J. J., y F. Salazar, (2009) La investigación educacional en Chile: Una aproximación bibliométrica no convencional. Documento de Trabajo. Santiago, Centro de Políticas Comparadas de Educación CPCE Número 1. Disponible en: http://www.cpce.cl/publicaciones/documentos-detrabajo/29-la-investigacion-educacional-en-chile-una-aproximacionbibliometrica-no-convencional [Recuperado el 15 de junio de 2010]

Bourdieu, P., (2003) «El oficio del científico: ciencia de la ciencia y reflexividad». Curso del Collège de France 2000-2001, Barcelona, Anagrama.

Centro de Investigación y Desarrollo de la Educación y Ministerio de Educación de Chile, (2007) Estado del arte de la investigación y desarrollo en educación en Chile. Santiago, Ministerio de Educación de Chile MINEDUC.

Cisternas, T., (2011) «La investigación sobre formación docente en Chile. Territorios explorados e inexplorados» en Calidad en la Educación. Número 35. Diciembre 2011, pp. 131-164.

Cochran-Smith, M. y K. Fries, (2005) «Researching teacher education in changing times: politics and paradigms» en AERA (Ed.), Studying teacher education: the report of the AERA Panel on Research and Teacher Education, Nueva Jersey, Lawrence Erlbaum Associates.

Flick, U., (2004) Introducción a la investigación cualitativa. Primera edición. Madrid, Morata, Fundación Paideia.

Glaser, B. G., (2002) Conceptualization: On Theory and Theorizing Using Grounded Theory. International Journal of Qualitative Methods, 1(2). Recuperado el 10 marzo 2010, de http://www.ualberta.ca/ ijqm/

Glaser, B. G., (2004) Remodeling Grounded Theory. Forum Qualitative Social Research, 5(2). Recuperado el 20 de mayo de 2009, de http://nbnresolving.de/urn:nbn:de:0114-fqs040245 
Grupo de Estudios Sociales de la Ciencia y la Tecnología, (2007) «La psicología social de la ciencia. Una revisión bibliográfica de su estado actual» en Athenea Digital. Número 11. Primavera 2007, pp. 161-208.

Íñiguez, L. y C. Pallí, (2002) «La psicología social de la ciencia: revisión y discusión de una nueva área de investigación» en Anales de psicología. Volumen 18, Número 1. Junio 2002, pp. 13-43.

Kennedy, M., (1995) «Research genres in teacher education» en Murray, F. (Ed.), Knowledge base in teacher education. Washington, D.C., McGraw Hill.

Knorr-Cetina, K., (2005) La fabricación del conocimiento. Un ensayo sobre el carácter constructivista y contextual de la ciencia. Buenos Aires, Universidad Nacional de Quilmes Editorial.

Latour, B. y S. Woolgar, (1995) Vida en laboratorios: la construcción de los hechos cientificos. Madrid, Alianza Universidad

Martin, O., (2003) Sociología de las ciencias. Buenos Aires, Ediciones Nueva Visión

Ramos, C., (2008) ¿Sistema, campo de lucha o red de traducciones y asociaciones? Tres modelos para investigar la ciencia social y un intento de integración. Persona y Sociedad. Volumen 22, Número 2. 1995, pp. 9-52.

Scott, K. W., \& D. M. Howell, (2008) Clarifying analysis and interpretation in grounded theory: Using a conditional relationship guide and reflective coding matrix. International Journal of Qualitative Methods, 7(2), 1-15.

Strauss, A., \& J. Corbin, (2002) Bases de la investigación cualitativa: técnicas y procedimientos para desarrollar la teoría fundamentada. Medellín: Universidad de Antioquía.

Tenti, F.E., (2001) «En casa de herrero cuchillo de palo: la producción y uso de conocimientos en el sistema educativo» paper presentado en el VI Congreso de Investigación Educativa, COMIE, Manzanillo, México, 2001.

Woolgar, S., (1991) Ciencia, abriendo la caja negra. Primera edición. Barcelona, Anthropos.

Zeichner, K., (1998) «Para além da divisão entre professor-pesquisador e pesquisador académico» en Gerladi, C.; Fiorentini, D. y E. Pereira (Eds.), Cartografia do trabalho docente: professor(a)-pesquisador(a). Campinas, Mercado de Letras.

Recibido: 03/04/2013

Aceptado: 29/05/2013 